\title{
MESOSCALE DIFFRACTIVE PHOTONICS IN GEOSCIENCES
}

\author{
I.V.Minin, O.V.Minin \\ Siberian State University of Geosystems and Technologies, 630108, Plahotnogo 10, Novosibirsk, Russia - prof.minin@gmail.com
}

KEY WORDS: atmosphere aerosol, particles, far field, near field, mesoscale, sensor.

\begin{abstract}
:
The scattered light by various dielectric particles in atmosphere give information about the type of molecules and particles and their location, which are important to definition of propagation limitations through atmospheric and space weather variations, crisis communications, etc. Although these investigations explain far field properties of disturbed radiations, the solution of the physical problem requires simulations of the interactions in near-field. It has been shown that strongly localized EM field near the surface of single dielectric particle may be form by non-spherical and non-symmetrical mesoscale particles both as in transmitting as in reflection mode. It was also shown that the main lobe is narrower in case of 3 cube chain than single cube in far field, but there are many side-scattering lobes. It was mentioned that unique advantages provided by mesoscale dielectric photonic crystal based particles with three spatial dimensions of arbitrary shape allow developing a new types of micro/nano-probes with subwavelength resolution for ultra compact spectrometer-free sensor for on board a spacecraft or a plane.
\end{abstract}

\section{INTRODUCTION}

Usually the light was used to illuminate a distant object, and when recording the direction to the object from the position of the observer it was the standard procedure of geodesy for centuries when generating image maps.

As it well known in the lidar (Ackermann, 1999) the light pulses are sent through the atmosphere. The returned light scattered by various air molecules and particles give information about the type of molecules and particles in the atmosphere and their location, which are important to definition of propagation limitations through atmospheric and space weather variations, crisis communications, etc. Meteorological lidars are also capable of monitoring a wide range of atmospheric parameters such as, for example, cloud particle properties (extinction coefficient, backscatter coefficient, depolarization), trace gas concentration, which are practical use of remote sensing images for mapping and cartographic production, etc.

Moreover these laser sensors (lidar and laser altimeters) on board a spacecraft or plane are used for obtaining highresolution maps through atmosphere with different applications, including geography, geology, seismology, forestry and many other areas (Carswell, 2011).

A major motivation for the problem of light interaction with dielectric particles can be found in the need to understand the atmospheric pertubations that affect free space traveling wave detection (van de Hulst, 1981) including astronomic observations and remote sensing. Aerosol particles modify the transfer of radiation in the atmosphere. Although these investigations were first undertaken to explain far field properties of disturbed radiations, the solution of the physical problem requires a simulations of the interactions in nearfield. On the other hand, a deeper understanding of the highly localized intensive optical flux near the surface of particle is nevertheless needed to fully exploit the potential performance of nano- and micro- dielectric mesoscale objects as diffractive photonic components in geosciences (Schmidt, 2015) at different wavebands and applications.

\section{NEAR-FIELD LIGHT INTERACTION WITH 3D PARTICLES}

\subsection{Single particle}

A first approximation consists in neglecting the coherent interactions of light with the nearby object, thereby reducing the problem to the interaction of light with a single scatterer. It could be noted that mesoscale particles pay an important role in the atmosphere optics effects (Riemer et al. 2003, Morgan et al. 1996).

As it well known, according to Lorenz-Mie theory, the optical field both inside and outside the low absorbing sphere subject to a light wave is characterized by the formation of focusing areas called inner and outer focuses. They result from the spherical particle surface curvature (R.K. Chang and Y.-Le Pan, 2008), which causes relevant deformations of the incident phase wave front. Thus, spherical microparticle acts as a refractive microlens focusing light wave within subwavelength volume (Ferrand et al, 2008).

The scattering of electromagnetic waves at nano- and microcylindrical and spherical particles by using different numerical techniques was the subject of intense investigations around the world. Investigations shown that strongly localized electromagnetic (EM) field near the surface of the particle is formed in the spatial region where the effects of near-field scattering (evanescent fields) play a significant role. Typically, this near-field region is located at the distances not exceeding several particle diameters and is characterized by marked contribution of the radial component of optical field (Heifetz et al. 2009). In turn, this condition imposes limitations on the range of dielectric particles sizes, so it must be about a few wavelengths and even equal to the radiation wavelength, i.e., have a mesoscale dimensions. So the specific value of the incident wavelength is not critical as long as the mesoscale conditions satisfied.

So far, the bulk of theoretical studies are devoted to the light strongly localized EM field (photonic jet) originated from microparticles with high degree of spatial symmetry like spheres, spheroids, cylinders, disks because of their unique capability to produce extreme spatial field localization. In the paper (Minin and Minin, 2014) the authors intend to fill this gap and report the brief review and results of investigations on localized EM field formation by the particles with more complicated non-spherical spatial shapes and even by nonsymmetric bodies. 


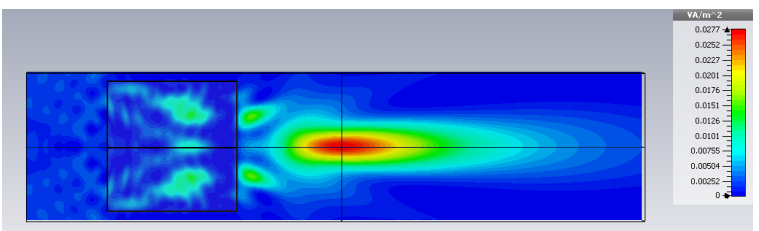

Fig.1. Example of near field intensity simulation for dielectric cuboid particle with dimensions of $4 \times 4 \times 4$ in wavelength.

It has been shown that strongly localized EM field may be form by non-spherical and non-symmetrical dielectric mesoscale particles both as in transmitting (Fig.1) as in reflection mode. It allows a better understanding of localized EM field properties and possible strategies for tailoring such beams in different practical applications (Minin and Minin, 2016).

\subsection{Array of particles}

Investigation of forward scattering radiation by linear chain of 3 dielectric cubic particles, with dimensions equal to the wavelength was done in the form of calculation experiment. The geometry of the problem is shown in the Fig.2. Simulation results of the radar cross-section in far field are shown in the Fig.3.

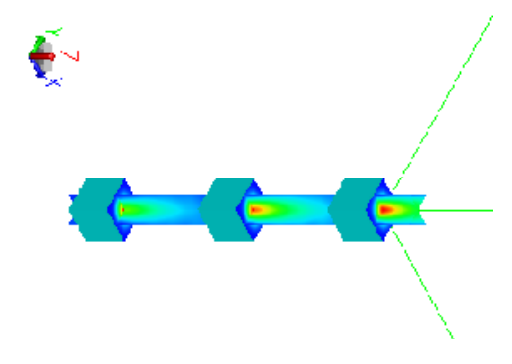

Fig.2. Linear chain of 3 mesoscale dielectric cube: $n=1.41$, $\mathrm{L}=\mathrm{H}=1 \lambda$, spacing $=2.5 \lambda$.

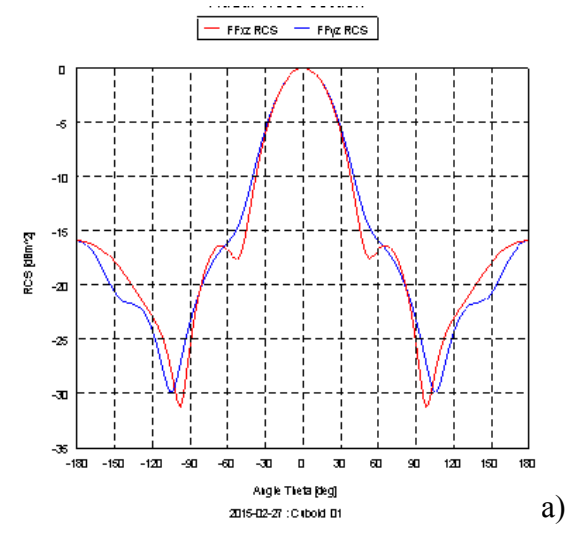

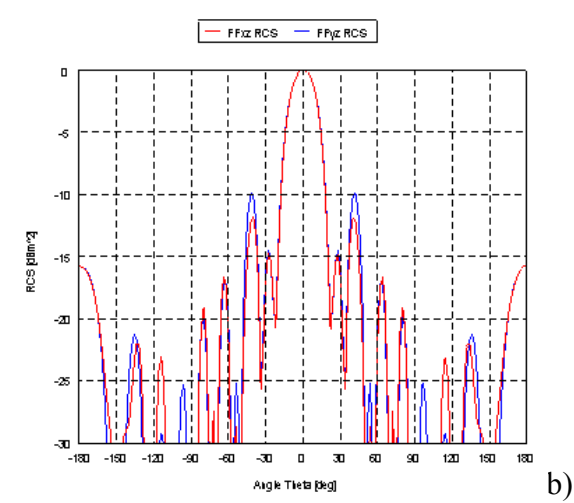

Fig.3. Radar cross-sections of single (a) and linear chain of 3 mesoscale cubes (b) in far field.

Simulations shown, that far-field radiation pattern for 3 cube chain is pretty different from single cube. The main lobe is narrower in case of 3 cube chain than single cube, but there are many side-scattering lobes. If we decrease the gap between the cubes we reduce the number of side scattering lobes (as more side lobes more loss of optical energy).

The theoretical demonstration of azimuthally symmetric, ultra-directional Kerker's-type far field scattering of a chain of dielectric cubes with high permittivity (more than 10) in visible and near-infrared regions via simultaneous excitation and interference of optically induced electric- and magneticresonances up to quadrupolar modes was discussed by Sikdar et al (Sikdar et al, 2015).

With lower permittivity (we have used particle with refractive index $\mathrm{n}=1.41$ ), the strength of both electric and magnetic dipoles of particle is reduced. However, it must be possible to find the wavelength where their strengths spectrally overlap and there is interference between them to give rise to unidirectionality. But the wavelength of such interfering conditions may be different from the wavelengths of resonance, in which case the scattering intensity will get lowered.

Also for the chain of cubic particles, the effect of an enhanced transmittance with increasing packing density of particles was observed.

\section{SPECTROSCOPY}

The use of small dielectric particles as near-field subdiffraction focusing systems provides highly compact and efficient systems that can furthermore utilize evanescent field contributions. This results in strongly localized electromagnetic fields providing a potential downscaling of classical optical and electromagnetic devices.

The latest advances in metamaterials and plasmonics allow providing new routes for light control and localization, with the aim to improve existing sensors and provide new avenues for high-accuracy sensing. A new class of compact mesoscale spectroscopy systems in which the three-dimensional shape of the electromagnetic field is engineered to induce qualitatively new physical effects, for example, in molecules is also envisioned (Minin and Minin, 2015; Minin and Minin, 2016). 


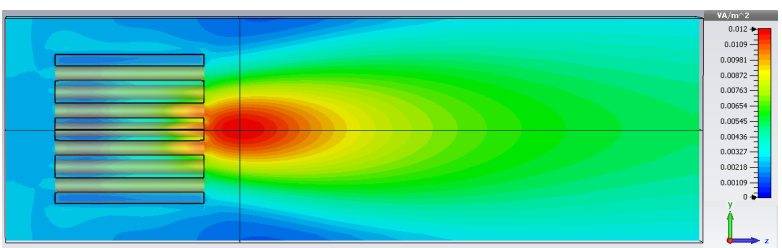

Fig.4. Simulation of metamaterial aided birefringent photonic jet for spectroscopic application.

For example, metamaterial aided (Fig.4) birefringent photonic jet (focusing devices) are novel objects with many unusual properties and, more importantly, they may demonstrate much broader spectrum of potential applications, including spectroscopy in comparison with the isotropic metamaterials.

It could be also noted that the unique advantages provided by mesoscale dielectric photonic crystal based particles with three spatial dimensions of arbitrary shape allow developing a new types of micro/nano-probes with subwavelength resolution for ultra compact spectrometer free sensor for on board a spacecraft or plane chemical, gas concentration and biochemical/biomedical sensing.

\section{CONCLUSION}

It has been shown that strongly localized EM field near the surface of single 3D dielectric particle may be form by nonspherical and non-symmetrical mesoscale particles both as in transmitting as in reflection mode. It was also shown that the main lobe is narrower in case of 3 cube chain than single cube in far field, but there are many side-scattering lobes. The unique advantages provided by mesoscale dielectric photonic crystal based particles with three spatial dimensions of arbitrary shape allow developing a new types of micro/nano-probes with subwavelength resolution for ultra compact spectrometer-free sensor for on board a spacecraft or a plane. It allow to conclude that a new physical direction called low dielectric permittivity photonics open a new horizon in different fields of physics.
F.Ackermann, 1999. Airborne laser scanning - present status and future expectations, Photogrammetry and Remote Sensing 54, pp. 64-67.

A. Carswell, 2011. Lidar Imagery - From Simple Snapshots to Mobile 3D Panoramas, in Photogrammetric Week'11, Ed. D. Fritsch, Wichmann, VDE Verlag, Berlin and Offenbach, pp. 3-14.

H. C. van de Hulst, 1981. Light Scattering by Small Particles, Dover Publication, Inc.

W.Schmidt, 2015. Photonics in Geosciences http://light2015blog.org/

N. Riemer, et al, 2003. Modeling aerosols on the mesoscale$\gamma$ : Treatment of soot aerosol and its radiative effects. $J$. of Geophysical Research, V. 108 (D19), p.4601.

M.Moran, R.A. Pielke, 1996. Evaluation of a mesoscale atmospheric dispersion modeling system with observation from the 1.980 great plains mesoscale tracer field experiment. PartII : dispersion simulations. Journal of Applied Meteorology. Boston. V.35 (3), pp. 308-329.

R.K. Chang and Y.-Le Pan, 2008. Linear and non-linear spectroscopy of microparticles: Basic principles, new techniques and promising applications. Faraday Discuss., v.137,pp. 9-36.

P. Ferrand et al, 2008. Direct imaging of photonic nanojets. Opt. Express, v.16, pp.6930-6940.

A. Heifetz et al, 2009. Photonic Nanojets. Comput. Theor. Nanosci. V.6, pp.1979-1984.

I.V. Minin and O.V. Minin, 2014. Photonics of isolated dielectric particles of arbitrary 3D shape - a new direction of optical information technologies. Vestn. NGU. Ser. Technol. V.4, pp.59-70 (in Russian).

D. Sikdar et al. 2015. Optically resonant magneto-electric cubic nanoantennas for ultra-directional light scattering. Journal of Applied Physics, v.117, pp.083101-083116.

I.V.Minin and O.V.Minin, 2015. Effect of EM strong localization in photonic crystal. - in $3{ }^{\text {rd }}$ Int. Conf. "Photonics of nano- and micro- structures" (FNMS-2015), Tomsk, 7-11 Sept., TUSUR.

\section{REFERENCES}

